\title{
Supersymmetrical Separation of Variables in Two-Dimensional Quantum Mechanics
}

\author{
Mikhail V. IOFFE \\ Saint-Petersburg State University, St.-Petersburg, 198504 Russia \\ E-mail: m.ioffe@pobox.spbu.ru \\ URL: http://hep.niif.spbu.ru/staff/ioffe_e.htm
}

Received August 24, 2010, in final form September 19, 2010; Published online September 24, 2010 doi:10.3842/SIGMA.2010.075

\begin{abstract}
Two different approaches are formulated to analyze two-dimensional quantum models which are not amenable to standard separation of variables. Both methods are essentially based on supersymmetrical second order intertwining relations and shape invariance two main ingredients of the supersymmetrical quantum mechanics. The first method explores the opportunity to separate variables in the supercharge, and it allows to find a part of spectrum of the Schrödinger Hamiltonian. The second method works when the standard separation of variables procedure can be applied for one of the partner Hamiltonians. Then the spectrum and wave functions of the second partner can be found. Both methods are illustrated by the example of two-dimensional generalization of Morse potential for different values of parameters.
\end{abstract}

Key words: supersymmetry; separation of variables; integrability; solvability

2010 Mathematics Subject Classification: 81Q60

\section{Introduction}

The exactly solvable models in quantum mechanics are of special interest during many years both by methodological and practical reasons. By now, the main achievements were related to one-dimensional Shrödinger equation. Indeed, a list of exactly solvable one-dimensional problems (Harmonic oscillator, Coulomb, Morse, Pöschl-Teller potentials etc.) was obtained by an algebraic procedure in the framework of factorization method [1] in the middle of last century. This method was reproduced rather recently in supersymmetrical quantum mechanics approach [2] initiated by the seminal papers of E. Witten [3]. More of that, this approach gave many new exactly solvable potentials which were obtained as superpartners of "old" exactly solvable models. It is necessary to mention also the important paper of L. Gendenstein [4], where the new fruitful notion of shape invariance was introduced. For the sake of truth, more than a century ago the so called Darboux transformation [5] for Sturm-Liouville equation was well known among mathematicians. Its application to a specific Schrödinger-like equation is actually equivalent $[6,7]$ to the factorization method.

The situation is much worse for two-dimensional quantum mechanics. The only regular method to solve analytically the Schrödinger equation is well known method of separation of variables [8]. This method replaces the two-dimensional problem by a pair of one-dimensional problems. It can be used for very restrictive class of models. Full classification of models which allowed separation of variables was given by L.P. Eisenhart [9]: four possibilities exist Cartesian, polar, elliptic and parabolic coordinates. The general form of potentials amenable to

${ }^{\star}$ This paper is a contribution to the Proceedings of the Workshop "Supersymmetric Quantum Mechanics and Spectral Design" (July 18-30, 2010, Benasque, Spain). The full collection is available at http://www.emis.de/journals/SIGMA/SUSYQM2010.html 
separation of variables is known explicitly up to arbitrary functions of one variable. And analytical solution is possible only if these functions belong to the list of exactly solvable potentials. All these Hamiltonians $H$ are integrable: the symmetry operator $R$ of second order in derivatives (in momenta) exists: $[H, R]=0$. Besides models amenable to separation of variables, the class of so called Calogero-like models [10] is known as well. They describe the specific forms of pairwise interaction of $N$ particles on a line, and they are solvable by means of special transformation of variables which leads to a separation of variables. The problem is in analogous state for higher dimensions of space.

Intermediate class of models - quasi-exactly-solvable (QES) potentials (or, partially solvable) - became interesting during last years. This notion concerns models for which only a part of spectrum and corresponding wave functions can be found analytically. In one-dimensional quantum mechanics a lot of such models were built with some hidden algebraic structure [11]. The supersymmetrical approach also gave some new QES potentials [12].

Thus, the search of new approaches to solution of nontrivial two-dimensional quantum mechanical models seems to be of current importance. It was already mentioned above that supersymmetrical quantum mechanics provides both new ways to derive some old results and interesting method to obtain new ones. In this paper we shall focus on the case of two-dimensional Schrödinger equation. Namely, we shall present two procedures of using of the supersymmetrical intertwining relations with supercharges of second order in derivatives as procedures of SUSY-separation of variables.

In Section 2 the general form of two-dimensional supersymmetrical quantum mechanics with second order supercharges will be formulated. Section 3 presents the first procedure of SUSY separation of variables where variables are separated in the supercharge. It leads to QES models, and the specific model of two-dimensional Morse potential illustrates this method. In Section 4 the second procedure of SUSY separation of variables is given where variables are separated in one of partner Hamiltonians. In the case of the same Morse model, but with particular values of parameter, it allows to solve the model completely, i.e. to find analytically the whole spectrum and all wave functions.

\section{Two-dimensional SUSY quantum mechanics}

Direct generalization of one-dimensional Witten's SUSY quantum mechanics to the arbitrary dimensionality $d$ of space was formulated in $[6,13]$. The Superhamiltonian included $(d+1)$ matrix components of different matrix dimensionality, and these components are intertwined by components of supercharge - operators linear in derivatives. In particular, in the case of $d=2$ two scalar Hamiltonians and a $2 \times 2$ matrix Hamiltonian are intertwined

$$
H^{(0)} \underset{q_{i}^{ \pm}}{\Longleftrightarrow} H_{i k} \underset{p_{i}^{\mp}}{\Longleftrightarrow} \widetilde{H}^{(0)}
$$

where

$$
q_{i}^{ \pm}=\mp \partial_{i}+\left(\partial_{i} W(\vec{x})\right), \quad p_{i}^{ \pm}=\varepsilon_{i k} q_{k}^{\mp}, \quad H^{(0)} q_{i}^{+}=q_{k}^{+} H_{k i}^{(1)}, \quad \widetilde{H}^{(0)} p_{i}^{+}=p_{k}^{+} H_{k i}^{(1)} .
$$

Some physical problems were considered in this framework. For example, the spectrum of the Pauli operator describing spin 1/2 fermion in the external electrostatic and magnetic field was investigated $[14,15,16]$. However, the following natural question arises: is it possible to avoid matrix Hamiltonians from the scheme? Any attempt to intertwine two scalar Hamiltonians by means of first order operators leads to potentials with standard separation of variables [17] which are not interesting for us here. 
The nontrivial way to avoid matrix Hamiltonians lies in the framework of polynomial SUSY. The latter was used for the first time $[18,7]$ in one-dimensional SUSY quantum mechanics. In two-dimensional context it was proposed in [19], where a pair of scalar two-dimensional Hamiltonians $H^{(0)}, H^{(1)}$ was intertwined by second order operators $Q^{ \pm}$

$$
H^{(0)} Q^{+}=Q^{+} H^{(1)}, \quad Q^{-} H^{(0)}=H^{(1)} Q^{-},
$$

where the Hamiltonians have the Schrödinger form

$$
H^{(0,1)}=-\partial_{i}^{2}+V^{(0,1)}(\vec{x}) .
$$

As for the intertwining supercharges $Q^{ \pm}$, the first naive idea is to choose reducible (factorized) supercharge $Q^{+}=q_{i}^{+} \widetilde{q}_{i}^{-}$

$$
\begin{aligned}
& q_{i}^{ \pm} \quad p_{i}^{\mp} \\
& H^{(0)} \Longleftrightarrow H_{i k} \Longleftrightarrow \widetilde{H}^{(0)} \\
& \| \\
& H^{(1)} \underset{\widetilde{q}_{i}^{ \pm}}{\Longleftrightarrow} H_{i k} \underset{\widetilde{p}_{i}^{\mp}}{\Longleftrightarrow} \widetilde{H}^{(1)}
\end{aligned}
$$

It is too naive [19], since this construction leads to Hamiltonians amenable to separation of variables in polar coordinates

$$
V(\vec{x})=a^{2} \rho^{2}+\frac{1}{\rho^{2}} F(\varphi) .
$$

The second idea is to choose $Q^{+}=q_{i}^{+} U_{i k} \widetilde{q}_{k}^{-}$with some unitary twist by constant matrix $U_{i k}$

$$
\begin{aligned}
& q_{i}^{ \pm} \quad p_{i}^{\mp} \\
& H^{(0)} \Longleftrightarrow H_{i k} \Longleftrightarrow \widetilde{H}^{(0)} \\
& \| \\
& H^{(1)} \underset{\widetilde{q}_{i}^{ \pm}}{\Longleftrightarrow} U_{i l} H_{l m} U_{m k}^{\dagger} \underset{\widetilde{p}_{i}^{\mp}}{\Longleftrightarrow} \widetilde{H}^{(1)}
\end{aligned}
$$

Some QES models were obtained by this trick [20].

The most general form of second order supercharges

$$
Q^{+}=g_{i k}(\vec{x}) \partial_{i} \partial_{k}+C_{i}(\vec{x}) \partial_{i}+B(\vec{x}), \quad Q^{-} \equiv\left(Q^{+}\right)^{\dagger}
$$

leads to a complicate system of nonlinear second order differential equations for functions $g_{i k}$, $C_{i}, B$, and potentials $V^{(0,1)}(\vec{x})$. Its general solution is impossible, but some particular solutions were found $[19,21]$. The simplest choice $g_{i k}(\vec{x})=\delta_{i k}$ gives the separation of variables in polar coordinates. The Lorentz form $g_{i k}(\vec{x})=\operatorname{diag}(1,-1)$ does not lead to separation of variables, and some particular solutions were found [19].

Here we focus on $g_{i k}(\vec{x})=\operatorname{diag}(1,-1)$. In this case, the system is simplified essentially. New variables $x_{ \pm}=x_{1} \pm x_{2}$ are useful together with $x_{1}, x_{2}$. Using the intertwining relations, one can prove that new functions $C_{ \pm}$depend on one variable only

$$
C_{+} \equiv C_{1}-C_{2}=C_{+}\left(x_{+}\right), \quad C_{-} \equiv C_{1}+C_{2}=C_{-}\left(x_{-}\right), \quad x_{ \pm}=x_{1} \pm x_{2} .
$$

The general solution for Lorentz metric can be provided by solving the only equation

$$
\partial_{-}\left(C_{-} F\right)=-\partial_{+}\left(C_{+} F\right),
$$


where new useful function is $F=F_{1}\left(x_{+}+x_{-}\right)+F_{2}\left(x_{+}-x_{-}\right)$. Thus, the equation is the functional differential equation, and no regular procedure of its solution is known.

The required potentials $V^{(0,1)}(\vec{x})$ and the function $B(\vec{x})$ are expressed in terms of $C_{ \pm}$and $F_{1,2}$

$$
\begin{aligned}
& V^{(0,1)}= \pm \frac{1}{2}\left(C_{+}^{\prime}+C_{-}^{\prime}\right)+\frac{1}{8}\left(C_{+}^{2}+C_{-}^{2}\right)+\frac{1}{4}\left(F_{2}\left(x_{+}-x_{-}\right)-F_{1}\left(x_{+}+x_{-}\right)\right), \\
& B=\frac{1}{4}\left(C_{+} C_{-}+F_{1}\left(x_{+}+x_{-}\right)+F_{2}\left(x_{+}-x_{-}\right)\right) .
\end{aligned}
$$

A variety of such pairs of potentials was found in [19].

\section{SUSY-separation of variables I: QES models}

The first variant of SUSY-separation of variables is realized when the Hamiltonian $H$ does not allow standard separation of variables, but the supercharge $Q^{+}$does allow $[22,23]$. The general scheme is the following. Let's suppose that we know zero modes of $Q^{+}$

$$
Q^{+} \Omega_{n}(\vec{x})=0, \quad n=0,1, \ldots, N, \quad Q^{+} \vec{\Omega}(\vec{x})=0 .
$$

The intertwining relation

$$
H^{(0)} Q^{+}=Q^{+} H^{(1)}
$$

obey the important property: the space of zero modes is closed under the action of $H^{(1)}$ :

$$
H^{(1)} \vec{\Omega}(\vec{x})=\hat{C} \vec{\Omega}(\vec{x}) .
$$

If the matrix $\hat{C}$ is known, and if it can be diagonalized

$$
\hat{B} \hat{C}=\hat{\Lambda} \hat{B}, \quad \hat{\Lambda}=\operatorname{diag}\left(\lambda_{0}, \lambda_{1}, \ldots, \lambda_{N}\right),
$$

the eigenvalues of $H^{(1)}$ can be found algebraically

$$
H^{(1)}(\hat{B} \vec{\Omega}(\vec{x}))=\hat{\Lambda}(\hat{B} \vec{\Omega}(\vec{x})) .
$$

Thus, for realization of this scheme we need

- to find zero modes $\Omega_{n}(\vec{x})$;

- to find constant matrix $B$, such that $\hat{B} \hat{C}=\hat{\Lambda} \hat{B}$.

As for zero modes, they can be obtained by using the special similarity transformation (not unitary!), which removes the terms linear in derivatives from $Q^{+}$

$$
\begin{aligned}
& q^{+}=e^{-\chi(\vec{x})} Q^{+} e^{+\chi(\vec{x})}=\partial_{1}^{2}-\partial_{2}^{2}+\frac{1}{4}\left(F_{1}\left(2 x_{1}\right)+F_{2}\left(2 x_{2}\right)\right), \\
& \chi(\vec{x})=-\frac{1}{4}\left(\int C_{+}\left(x_{+}\right) d x_{+}+\int C_{-}\left(x_{-}\right) d x_{-}\right) .
\end{aligned}
$$

Now, $q^{+}$allows separation of variables for arbitrary solution of intertwining relations, and we obtain the first variant of new procedure - SUSY-separation of variables. Similarly to the conventional separation of variables, separation of variables in the operator $q^{+}$itself does not guarantee solvability of the problem.

The next task is to solve two one-dimensional problems

$$
\begin{aligned}
& \left(-\partial_{1}^{2}-\frac{1}{4} F_{1}\left(2 x_{1}\right)\right) \eta_{n}\left(x_{1}\right)=\epsilon_{n} \eta_{n}\left(x_{1}\right), \\
& \left(-\partial_{2}^{2}+\frac{1}{4} F_{2}\left(2 x_{2}\right)\right) \rho_{n}\left(x_{2}\right)=\epsilon_{n} \rho_{n}\left(x_{2}\right) .
\end{aligned}
$$

Three remarks are appropriate now. 
Remark 1. The same similarity transformation of $H^{(1)}$ does not lead to operator amenable to separation of variables.

Remark 2. The normalizability of $\Omega_{n}$ has to be studied attentively due to non-unitarity of the similarity transformation.

Remark 3. We have no reasons to expect exact solvability of the model, but quasi-exactsolvability can be predicted.

As for the matrix $\hat{B}$, it must be found by some specific procedure. Such procedure was used in example which will be presented below.

In principle, the first scheme of SUSY-separation of variables can be used for arbitrary models satisfying intertwining relations by supercharges with Lorentz metrics. The list of solutions of intertwining relations is already rather long, and it may increase in future. The main obstacle is analytical solvability of one-dimensional equations, obtained after separation of variables in the operator $q^{+}$.

Below we describe briefly such a model which can be considered as the generalized twodimensional Morse potential

$$
\begin{aligned}
& C_{+}=4 a \alpha, \quad C_{-}=4 a \alpha \operatorname{coth} \frac{\alpha x_{-}}{2}, \\
& f_{i}\left(x_{i}\right) \equiv \frac{1}{4} F_{i}\left(2 x_{i}\right)=-A\left(e^{-2 \alpha x_{i}}-2 e^{-\alpha x_{i}}\right), \quad i=1,2, \\
& V^{(0),(1)}=\alpha^{2} a(2 a \mp 1) \sinh ^{-2}\left(\frac{\alpha x_{-}}{2}\right)+4 a^{2} \alpha^{2}+A\left[e^{-2 \alpha x_{1}}-2 e^{-\alpha x_{1}}+e^{-2 \alpha x_{2}}-2 e^{-\alpha x_{2}}\right],
\end{aligned}
$$

where $A>0, \alpha>0, a$ is real.

To explain the name, we present the potential in the form

$$
V(\vec{x})=V_{\text {Morse }}\left(x_{1}\right)+V_{\text {Morse }}\left(x_{2}\right)+v\left(x_{1}, x_{2}\right),
$$

where first two terms are just one-dimensional Morse potentials, and the last term mixes variables $x_{1}, x_{2}$.

The solutions of one-dimensional Schrödinger equations are well known [24], and the zero modes can be written $[22,23]$ as

$$
\begin{aligned}
& \Omega_{n}(\vec{x})=\left(\frac{\alpha}{\sqrt{A}} \frac{\xi_{1} \xi_{2}}{\left|\xi_{2}-\xi_{1}\right|}\right)^{2 a} \exp \left(-\frac{\xi_{1}+\xi_{2}}{2}\right)\left(\xi_{1} \xi_{2}\right)^{s_{n}} F\left(-n, 2 s_{n}+1 ; \xi_{1}\right) F\left(-n, 2 s_{n}+1 ; \xi_{2}\right), \\
& \xi_{i} \equiv \frac{2 \sqrt{A}}{\alpha} \exp \left(-\alpha x_{i}\right), \quad s_{n}=\frac{\sqrt{A}}{\alpha}-n-\frac{1}{2}>0 .
\end{aligned}
$$

The conditions of normalizability and of absence of the "fall to the center" are

$$
a \in\left(-\infty,-\frac{1}{4}-\frac{1}{4 \sqrt{2}}\right), \quad s_{n}=\frac{\sqrt{A}}{\alpha}-n-\frac{1}{2}>-2 a>0
$$

To obtain the matrix $\hat{C}$ explicitly, one must act by $H^{(1)}$ on $\Omega_{n}$. The matrix turns out to be triangular, and therefore, the energy eigenvalues coincide with its diagonal elements

$$
E_{k}=c_{k k}=-2\left(2 a \alpha^{2} s_{k}-\epsilon_{k}\right) .
$$

To find a variety of wave functions is a more difficult task. For that it is necessary to find all elements of $\hat{C}$ and all elements of matrix $\hat{B}$. The recurrent procedure for the case of twodimensional Morse potential was given in $[22,23]$. This variety can be enlarged by means of shape invariance property [25] of the model

$$
H^{(0)}(\vec{x} ; a)=H^{(1)}(\vec{x} ; \tilde{a})+\mathcal{R}(a), \quad \tilde{a}=a-1 / 2, \quad \mathcal{R}(a)=\alpha^{2}(4 a-1) .
$$


Similarly to one-dimensional shape invariance, each wave function constructed by SUSY-separation of variables leads to a set of additional wave functions

$$
\begin{aligned}
H^{(0)}(a) & {\left[Q^{-}(a) Q^{-}\left(a-\frac{1}{2}\right) \cdots Q^{-}\left(a-\frac{M-1}{2}\right) \Psi\left(a-\frac{M}{2}\right)\right] } \\
= & \left(E_{0}\left(a-\frac{M}{2}\right)+\mathcal{R}\left(a-\frac{M-1}{2}\right)+\cdots+\mathcal{R}(a)\right) \\
& \times\left[Q^{-}(a) Q^{-}\left(a-\frac{1}{2}\right) \cdots Q^{-}\left(a-\frac{M-1}{2}\right) \Psi\left(a-\frac{M}{2}\right)\right] .
\end{aligned}
$$

Analogous approach works for the two-dimensional generalization of Pöschl-Teller model [20] and for some two-dimensional periodic potentials [26].

\section{SUSY-separation of variables II: exact solvability}

Among all known solutions of two-dimensional intertwining relations with second order supercharges a subclass exists [27], where one of intertwined Hamiltonians is amenable to standard separation of variables due to specific choice of parameters of the model. Its superpartner still does not allow separation of variables.

The scheme will be described below for the same specific model which is two-dimensional generalization of Morse potential

$$
V^{(0),(1)}=\alpha^{2} a(2 a \mp 1) \sinh ^{-2}\left(\frac{\alpha x_{-}}{2}\right)+4 a^{2} \alpha^{2}+A\left[e^{-2 \alpha x_{1}}-2 e^{-\alpha x_{1}}+e^{-2 \alpha x_{2}}-2 e^{-\alpha x_{2}}\right] .
$$

Let's choose $a_{0}=-1 / 2$ in order to vanish the mixed term in $V^{(1)}$. Then $H^{(1)}$ allows the conventional separation of variables. Moreover, after separation of variables each of obtained one-dimensional problems is exactly solvable. We met just this one-dimensional problem above in a different context.

The discrete spectrum of this one-dimensional model is

$$
\epsilon_{n}=-\alpha^{2} s_{n}^{2}, \quad s_{n} \equiv \frac{\sqrt{A}}{\alpha}-n-\frac{1}{2}>0, \quad n=0,1,2, \ldots
$$

Wave functions are expressed in terms of degenerate hypergeometric functions

$$
\eta_{n}\left(x_{i}\right)=\exp \left(-\frac{\xi_{i}}{2}\right)\left(\xi_{i}\right)^{s_{n}} F\left(-n, 2 s_{n}+1 ; \xi_{i}\right), \quad \xi_{i} \equiv \frac{2 \sqrt{A}}{\alpha} \exp \left(-\alpha x_{i}\right)
$$

Due to separation of variables, the two-dimensional problem with $H^{(1)}(\vec{x})$ is exactly solvable. Its energy eigenvalues are

$$
E_{n, m}=E_{m, n}=\epsilon_{n}+\epsilon_{m},
$$

being two-fold degenerate for $n \neq m$. The corresponding eigenfunctions can be chosen as symmetric or (for $n \neq m$ ) antisymmetric combinations

$$
\Psi_{E_{n, m} S, A}^{(1)}(\vec{x})=\eta_{n}\left(x_{1}\right) \eta_{m}\left(x_{2}\right) \pm \eta_{m}\left(x_{1}\right) \eta_{n}\left(x_{2}\right) .
$$

Our aim here is to solve completely the problem for $H^{(0)}(\vec{x})$ with $a_{0}=-1 / 2$. The main tool is again the SUSY intertwining relations, i.e. isospectrality of $H^{(0)}$ and $H^{(1)}$ but up to zero modes and singular properties of $Q^{ \pm}$. In general, we may expect three kinds of levels of $H^{(0)}(\vec{x})$ : 
(i) The levels, which coincide with $E_{n m}$. Their wave functions can be obtained from $\Psi^{(1)}$ by means of $Q^{+}$.

(ii) The levels, which were absent in the spectrum of $H^{(1)}(\vec{x})$, if some wave functions of $H^{(0)}(\vec{x})$ are simultaneously the zero modes of $Q^{-}$. Then the second intertwining relation would not give any partner state among bound states of $H^{(1)}(\vec{x})$.

(iii) The levels, which were also absent in the spectrum of $H^{(1)}(\vec{x})$, if some wave functions of $H^{(0)}(\vec{x})$ become nonnormalizable after action of operator $Q^{-}$.

We have to analyze these three classes of possible bound states of $H^{(0)}$ one after another.

(i) The first SUSY intertwining relation gives the two-fold degenerate wave functions of $H^{(0)}$ with energies $E_{n m}: \Psi_{E_{n m}}^{(0)}=Q^{+} \Psi_{E_{n m}}^{(1)}$. But $Q^{+}$includes singularity on the line $x_{1}=x_{2}$, therefore the normalizability of $\Psi_{E_{n, m}}^{(0)}$ depends crucially on the behavior of $\Psi_{E_{n, m}}^{(1)}$ on the line $\xi_{1}=\xi_{2}$. One can check that only antisymmetric functions $\Psi^{(1)}$ survive, i.e. only symmetric $\Psi^{(0)}$ survive. This fact can be demonstrated [27] both by direct calculation and by indirect method - by means of symmetry operator $R^{(0)}$.

The indirect method explores that the symmetry operator $R^{(0)}=Q^{-} Q^{+}$for $a_{0}=-1 / 2$ can be written in terms of one-dimensional Morse Hamiltonians $h_{1}\left(x_{1}\right), h_{2}\left(x_{2}\right)$

$$
R^{(0)}=\left(h_{1}\left(x_{1}\right)-h_{2}\left(x_{2}\right)\right)^{2}+2 \alpha^{2}\left(h_{1}\left(x_{1}\right)+h_{2}\left(x_{2}\right)\right)+\alpha^{4} .
$$

Therefore,

$$
R^{(0)} \Psi_{E_{n, m}}^{(0) A}(\vec{x})=r_{n, m} \Psi_{E_{n, m}}^{A}(\vec{x}), \quad r_{n, m}=\alpha^{4}\left[(n-m)^{2}-1\right]\left[\left(s_{n}+s_{m}\right)^{2}-1\right],
$$

and

$$
\left\|\Psi_{E_{n, m}}^{(1) S}\right\|^{2}=\left\langle\Psi_{E_{n, m}}^{(0) A}\left|Q^{-} Q^{+}\right| \Psi_{E_{n, m}}^{(0) A}\right\rangle=r_{n, m}\left\|\Psi_{E_{n, m}}^{(0) A}\right\|^{2}
$$

For $n=m$, wave functions $\Psi_{E_{n, n}}^{(0) S}$ vanish identically by trivial reasons. It is clear now that wave functions $\Psi_{E_{n, n \pm 1}}^{(0) S}$ also vanish. For all other $n, m$, functions $\Psi_{E_{n, m}}^{(0) S}$ have positive and finite norm, and there is no degeneracy of these levels.

(ii) These possible bound states of $H^{(0)}$ are the normalizable zero modes of $Q^{-}$. The variety of such zero modes is known from [22]: they exist only for positive values of $a$

$$
a \in\left(\frac{1}{4}+\frac{1}{4 \sqrt{2}},+\infty\right)
$$

which does not contain the value $a_{0}=-1 / 2$. Thus, no normalizable bound states of this class exist for $H^{(0)}$.

(iii) We have to study an opportunity that $Q^{-}$destroys normalizability of some eigenfunctions of $H^{(0)}$. It could occur due to singular character of $Q^{-}$at $x_{1}=x_{2}$. The analysis was performed [27] in suitable coordinates. It shows that $Q^{-}$is not able to transform normalizable wave function to nonnormalizable. Therefore, the third class of possible wave functions $H^{(0)}$ does not exist too.

Summing up, the spectrum of $H^{(0)}$ with $a_{0}=-1 / 2$ consists only of the bound states with energies $E_{n m}$ for $|n-m|>1$. This spectrum is bounded from above by the condition of positivity of $s_{n}, s_{m}: n, m<\sqrt{A} / \alpha-1 / 2$. The corresponding wave functions are obtained analytically [27].

The results above can be expanded to the whole hierarchy of Morse potentials with $a_{k}=$ $-(k+1) / 2$ with $k=0,1, \ldots$ by means of shape invariance property. Let's denote elements of 
the hierarchy as $H^{(0)}\left(\vec{x} ; a_{k}\right), H^{(1)}\left(\vec{x} ; a_{k}\right)$. All these Hamiltonians are also exactly solvable due to shape invariance of the model

$$
H^{(0)}\left(\vec{x} ; a_{k-1}\right)=H^{(1)}\left(\vec{x} ; a_{k}\right), \quad k=1,2, \ldots
$$

This means that the following chain (hierarchy) of Hamiltonians can be built

$$
\begin{aligned}
H^{(1)}\left(\vec{x} ; a_{0}\right) \div H^{(0)}\left(\vec{x} ; a_{0}\right) & =H^{(1)}\left(\vec{x} ; a_{1}\right) \div H^{(0)}\left(\vec{x} ; a_{1}\right)=\cdots \div H^{(1)}\left(\vec{x} ; a_{k-1}\right) \\
& =H^{(0)}\left(\vec{x} ; a_{k}\right) \div H^{(0)}\left(\vec{x} ; a_{k}\right),
\end{aligned}
$$

where the sign $\div$ denotes intertwining by $Q^{ \pm}\left(a_{i}\right)$.

In the general case, the functions

$$
\Psi_{E_{n, m}}^{(0)}\left(\vec{x} ; a_{k}\right)=Q^{+}\left(a_{k}\right) \Psi_{E_{n, m}}^{(1)}\left(\vec{x} ; a_{k}\right)=Q^{+}\left(a_{k}\right) Q^{+}\left(a_{k-1}\right) \cdots Q^{+}\left(a_{0}\right) \Psi_{E_{n, m}}^{(1) A}\left(\vec{x} ; a_{0}\right)
$$

(if normalizable) are the wave functions of $H^{(0)}\left(\vec{x} ; a_{k}\right)$ with energies $E_{n, m}=-\alpha^{2}\left(s_{n}^{2}+s_{m}^{2}\right)$. The symmetries of wave functions alternate and depend on the length of chain. This is true but up to zero modes of operators $Q^{+}$.

It is necessary to keep under the control normalizability of $\Psi$ and zero modes of $Q^{+}$. This control is performed algebraically by means of identity, which must be fulfilled up to a function of $H$

$$
R^{(1)}\left(a_{k}\right)=R^{(0)}\left(a_{k-1}\right) .
$$

Actually, the following equation holds:

$$
Q^{-}\left(a_{k}\right) Q^{+}\left(a_{k}\right)=Q^{+}\left(a_{k-1}\right) Q^{-}\left(a_{k-1}\right)+\alpha^{2}(2 k+1)\left[2 H^{(0)}\left(\vec{x} ; a_{k-1}\right)+\alpha^{2}\left(2 k^{2}+2 k+1\right)\right] .
$$

These relations allowed to evaluate the norms of wave functions. The result is the following. The spectra of Hamiltonians $H^{(0)}\left(\vec{x} ; a_{k}\right)$ are not degenerate. They consist of the bound states with energies $E_{n, m}$, with indices $|n-m|>k+2$, and their wave functions $\Psi_{E_{n, m}}^{(0)}\left(\vec{x} ; a_{k}\right)$ were given analytically above.

\section{Acknowledgements}

I am very grateful to A.A. Andrianov, F. Cannata and D.N. Nishnianidze for fruitful collaboration and many useful discussions. The work was partially supported by the RFFI grant 09-01-00145-a.

\section{References}

[1] Schrödinger E., A method of determining quantum-mechanical eigenvalues and eigenfunctions, Proc. Roy. Irish Acad. Sect. A 46 (1940), 9-16.

Schrödinger E., Further studies on solving eigenvalue problems by factorization, Proc. Roy. Irish Acad. Sect. A 46 (1940), 183-206.

Schrödinger E., The factorization of the hypergeometric equation, Proc. Roy. Irish Acad. Sect. A 47 (1941), $53-54$.

Infeld L., Hull T.E., The factorization method, Rev. Modern Phys. 23 (1951), 21-68.

Dabrowska J.W., Khare A., Sukhatme U., Explicit wavefunctions for shape-invariant potentials by operator techniques, J. Phys. A: Math. Gen. 21 (1988), L195-L200.

[2] Cooper F., Khare A., Sukhatme U., Supersymmetry and quantum mechanics, Phys. Rep. 251 (1995), 267385, hep-th/9405029.

Junker G., Supersymmetrical methods in quantum and statistical physics, Texts and Monographs in Physics, Springer-Verlag, Berlin, 1996.

Bagchi B.K., Supersymmetry in quantum and classical mechanics, Chapman \& Hall/CRC Monographs and Surveys in Pure and Applied Mathematics, Vol. 116, Chapman \& Hall/CRC, Boca Raton, FL, 2001. 
[3] Witten E., Dynamical breaking of supersymmetry, Nuclear Phys. B 185 (1981), 513-554.

[4] Gendenshtein L.E., Derivation of exact spectra of the Schrödinger equation by means of SUSY, JETP Lett. 38 (1983), 356-359.

[5] Darboux G., Sur un proposition relative aux equations lineares, C. R. Acad. Sci. Paris 94 (1882), $1456-1459$.

[6] Andrianov A.A., Borisov N.V., Ioffe M.V., Quantum systems with identical energy spectra, JETP Lett. 39 (1984), 93-97.

Andrianov A.A., Borisov N.V., Ioffe M.V., Factorization method and Darboux transformation for multidimensional Hamiltonians, Theoret. and Math. Phys. 61 (1984), 1078-1088.

Andrianov A.A., Borisov N.V., Ioffe M.V., The factorization method and quantum systems with equivalent energy spectra, Supersymmetic equivalent quantum systems, Phys. Lett. A 105 (1984), 19-22.

[7] Bagrov V.G., Samsonov B.F., Darboux transformation, factorization and supersymmetry in one-dimensional quantum mechanics, Theoret. and Math. Phys. 104 (1995), 1051-1060.

[8] Miller W. Jr., Symmetry and separation of variables, Encyclopedia of Mathematics and its Applications, Vol. 4, Addison-Wesley Publishing Co., Reading, Mass. - London - Amsterdam, 1977.

[9] Eisenhart L.P., Enumeration of potentials for which one-particle Schrödinger equations are separable, Phys. Rev. 74 (1948), 87-89.

[10] Calogero F., Solution of one-dimensional $N$-body problems with quadratic and/or inversely quadratic pair potential, J. Math. Phys. 12 (1971), 419-436.

[11] Turbiner A.V., Quasi-exactly-solvable problems and sl(2) algebra, Comm. Math. Phys. 118 (1988), $467-474$. Ushveridze A.G., Quasi-exactly solvable models in quantum mechanics, Soviet J. Particles and Nuclei 20 (1989), 504-528.

[12] Cannata F., Ioffe M.V., Roychoudhury R., Roy P., A new class of PT-symmetric Hamiltonians with real spectra, Phys. Lett. A 281 (2001), 305-310, quant-ph/0011089.

Debergh N., Van Den Bossche B., Samsonov B.F., Darboux transformations for quasi-exactly solvable Hamiltonians, Internat. J. Modern Phys. A 17 (2002), 1577-1587, quant-ph/0201105.

[13] Andrianov A.A., Borisov N.V., Ioffe M.V., Eides M.I., Supersymmetric mechanics: a new look at the equivalence of quantum systems, Theoret. and Math. Phys. 61 (1984), 965-972.

Andrianov A.A., Borisov N.V., Eides M.I., Ioffe M.V., Supersymmetric origin of equivalent quantum systems, Phys. Lett. A 109 (1985), 143-148.

[14] Andrianov A.A., Ioffe M.V., Pauli fermions as components of $D=2$ supersymmetrical quantum mechanics, Phys. Lett. B 205 (1988), 507-510.

[15] Ioffe M.V., Neelov A.I., Pauli equation and the method of supersymmetric factorization, J. Phys. A: Math. Gen. 36 (2003), 2493-2506, hep-th/0302004.

[16] Ioffe M.V., Kuru Ş., Negro J., Nieto L.M., SUSY approach to Pauli Hamiltonians with an axial symmetry, J. Phys. A: Math. Gen. 39 (2006), 6987-7001, hep-th/0603005.

[17] Demircioğlu B., Kuru Ş., Önder M., Verçin A., Two families of superintegrable and isospectral potentials in two dimensions, J. Math. Phys. 43 (2002), 2133-2150, quant-ph/0201099.

[18] Andrianov A.A., Ioffe M.V., Spiridonov V.P., Higher-derivative supersymmetry and the Witten index, Phys. Lett. A 174 (1993), 273-279, hep-th/9303005.

Andrianov A.A., Ioffe M.V., Cannata F., Dedonder J.-P., Second order derivative supersymmetry, $q$ deformations and the scattering problem, Internat. J. Modern Phys. A 10 (1995), 2683-2702, hep-th/9404061.

[19] Andrianov A.A., Ioffe M.V., Nishnianidze D.N., Polynomial SUSY in quantum mechanics and second derivative Darboux transformations, Phys. Lett. A 201 (1995), 103-110, hep-th/9404120.

Andrianov A.A., Ioffe M.V., Nishnianidze D.N., Polynomial supersymmetry and dynamical symmetries in quantum mechanics, Theoret. and Math. Phys. 104 (1995), 1129-1140.

Cannata F., Ioffe M.V., Nishnianidze D.N., Double shape invariance of the two-dimensional singular Morse model, Phys. Lett. A 340 (2005), 31-36, hep-th/0504077.

[20] Ioffe M.V., Valinevich P.A., New two-dimensional quantum models partially solvable by the supersymmetrical approach, J. Phys. A: Math. Gen. 38 (2005), 2497-2510, hep-th/0409153.

Ioffe M.V., Mateos Guilarte J., Valinevich P.A., Two-dimensional supersymmetry: from SUSY quantum mechanics to integrable classical models, Ann. Physics 321 (2006), 2552-2565, hep-th/0603006.

[21] Ioffe M.V., Negro J., Nieto L.M., Nishnianidze D.N., New two-dimensional integrable quantum models from SUSY intertwining, J. Phys. A: Math. Gen. 39 (2006), 9297-9308. 
[22] Cannata F., Ioffe M.V., Nishnianidze D.N., New methods for the two-dimensional Schrödinger equation: SUSY-separation of variables and shape invariance, J. Phys. A: Math. Gen. 35 (2002), 1389-1404, hep-th/0201080.

[23] Ioffe M.V., A SUSY approach for investigation of two-dimensional quantum mechanical systems, J. Phys. A: Math. Gen. 37 (2004), 10363-10374, hep-th/0405241.

[24] Landau L., Lifshitz E., Quantum mechanics, Pergamon, London, 1965.

[25] Andrianov A.A., Cannata F., Ioffe M.V., Nishnianidze D.N., Systems with higher-order shape invariance: spectral and algebraic properties, Phys. Lett. A 266 (2000), 341-349, quant-ph/9902057.

Cannata F., Ioffe M.V., Nishnianidze D.N., Double shape invariance of the two-dimensional singular Morse model, Phys. Lett. A 340 (2005), 31-36, hep-th/0504077.

[26] Ioffe M.V., Mateos Guilarte J., Valinevich P.A., A class of partially solvable two-dimensional quantum models with periodic potentials, Nuclear Phys. B 790 (2008), 414-431, arXiv:0706.1344.

[27] Ioffe M.V., Nishnianidze D.N., Exact solvability of a two-dimensional real singular Morse potential, Phys. Rev. A 76 (2007), 052114, 5 pages, arXiv:0709.2960. 